\title{
ON NON-ABELIAN SCHUR GROUPS
}

\author{
ILYA PONOMARENKO AND ANDREY VASIL'EV
}

\begin{abstract}
A finite group $G$ is called Schur, if every Schur ring over $G$ is associated in a natural way with a regular subgroup of $\operatorname{Sym}(G)$ that is isomorphic to $G$. We prove that any nonabelian Schur group $G$ is metabelian and the number of distinct prime divisors of the order of $G$ does not exceed 7 .
\end{abstract}

\section{INTRODUCTION}

Let $G$ be a finite group. In what follows we are interested in a permutation group $\Gamma \leq \operatorname{Sym}(G)$ that contains a subgroup $G_{\text {right }}$ induced by the right multiplications in $G$. Denote by $\mathcal{S}$ the set of orbits of the stabilizer of the identity $e=e_{G}$ in $\Gamma$. Then one can form a $\mathbb{Z}$-submodule $\mathcal{A}=\mathcal{A}(\Gamma, G)$ of the group ring $\mathbb{Z} G$ :

$$
\mathcal{A}=\operatorname{Span}\{\underline{X}: X \in \mathcal{S}\}
$$

where $\underline{X}$ is the sum of elements of $X$ in the group ring. It was observed by Schur in 21 that this submodule is a subring of the latter ring. The idea of Schur was to study the group $\Gamma$ by means of the ring $\mathcal{A}$ rather than by means of the characters of $G$. In this way he was able to prove a generalization of the Burnside theorem on a permutation group with a regular cyclic $p$-subgroup (see [24, Theorem 25.3]).

Following Wielandt a subring $\mathcal{A}$ of the ring $\mathbb{Z} G$ is called a $S$ chur ring or $S$-ring over the group $G$ if there exists a partition $\mathcal{S}$ of this group such that equality (1) holds and

$$
\{e\} \in \mathcal{S} \text { and } \mathcal{S}^{-1}=\mathcal{S}
$$

where $\mathcal{S}^{-1}=\left\{X^{-1}: X \in \mathcal{S}\right\}$ with $X^{-1}=\left\{x^{-1}: x \in G\right\} 11$ It is easily seen that the ring $\mathcal{A}(\Gamma, G)$ defined in the above paragraph is an S-ring over $G$. In particular, so is the group ring itself: in this case $\Gamma=G_{\text {right }}$.

It should be mentioned that as Wielandt wrote in 25, p.54]: Schur had conjectured for a long time that every $S$-ring $\mathcal{A}$ over a group $G$ is determined by a suitable permutation group $\Gamma$; in our terms this means that $\mathcal{A}=\mathcal{A}(\Gamma, G)$. However, this proved to be false and first counterexamples were found by Wielandt in [24, Theorem 25.7]. In honour of this Schur's fallacy the S-rings arising from permutation groups were called schurian in [20. It was also proved there that any S-ring over a cyclic $p$-group, $p>3$, is Schurian. Thus such a group is an example of a Schur group in the sense of the following definition given there.

Date: 05.07 .14

The work was partially supported by RFBR, research projects No. 14-01-00156 (first author) and No. 13-01-00505 (second author).

${ }^{1}$ One can see that the partition $\mathcal{S}$ is uniquely determined. 
Definition 1.1. A finite group $G$ is called a Schur group, if every $S$-ring over $G$ is schurian.

The problem of determining all Schur groups was suggested by R.Pöschel who proved that a $p$-group, $p>3$, is Schur if and only if it is cyclic [20. Only about thirty years after, all cyclic Schur groups were classified in [9]. The techique developed there were used in [10] where it was proved that any abelian Schur group belongs to one of the several explicitly given families. It should be mentioned that in the proof of this result a knowledge of Schur groups of small order is used. In fact, all S-rings over an arbitrary group of order $\leq 47$ were enumerated by means of computer computations. It turned out that there are non-abelian Schur groups. However, except for the above result of Pöschel, no general results on these groups was known. One of the main results of this paper gives a result of this type.

Theorem 1.2. A simple group is Schur if and only if it is abelian. In particular, any Schur group is solvable.

The proof of Theorem 1.2 is based on the Thompson theorem on minimal simple groups, a detailed analyses of groups of small order (Section 3), dihedral groups (Section 5) and the groups $\mathrm{PSL}_{2}(q)$ (Lemma 6.1). Based on Theorem 1.2 and the above analyses, we apply standard group-theoretical arguments to obtain the second main result of the paper.

Theorem 1.3. Any Schur group $G$ is metabelian. Moreover,

(1) $|\pi(G)| \leq 7$ with equality only if $|G|$ is even product of seven primes,

(2) if a Hall $\{2,3\}$-subgroup of $G$ is cyclic, then $G$ is metacyclic.

Concerning permutation groups we refer to 24]. For the reader convenience we collect the basic facts on S-rings and Cayley schemes in Section 2 .

Notation. For a positive integer $n$ we denote by $C_{n}, D_{n}$ and $E_{n}$ the cyclic, dyhedral and elementary abelian groups of order $n$, and by $S_{n}$ and $A_{n}$ the symmetric and alternating groups on $n$ letters respectively

The quaternion group and generalized quaternion groups are denoted by $Q_{8}$ and $Q_{2^{k}}, k \geq 4$, respectively.

For $k \geq 4$ we set $S D_{2^{k}}=\left\langle a, b \mid a^{2^{k-1}}=b^{2}=1, a^{b}=a^{-1+2^{k-2}}\right\rangle$ to be the semidihedral group 3

For a prime $p$ and $k \geq 3$ we set $M_{p^{k}}=\left\langle a, b \mid a^{p^{k-1}}=b^{p}=1, a^{b}=a^{1+p^{k-2}}\right\rangle$, where $k>3$ if $p=2$.

We set $G_{16}=\left\langle a, b, c \mid a^{4}=b^{2}=c^{2}=[a, b]=[a, c]=1,[b, c]=a^{2}\right\rangle$ 自

For a group $G$ we set $e_{G}$ to be the identity of $G$.

For groups $H$ and $G$ we write $H \prec G$ if $H$ is isomorphic to a section of $G$, that is a group $A / B$ with $B \triangleleft A \leq G$.

The set of prime divisors of a group $G$ is denoted by $\pi(G)$.

The group ring of $G$ over integers is denoted by $\mathbb{Z} G$.

The sum of elements of a set $X \subset G$ in $\mathbb{Z} G$ is denoted by $\underline{X}$.

The symmetric group on a set $\Omega$ is denoted by $\operatorname{Sym}(\Omega)$.

The regular permutation group which is induced by right (resp. left) multiplications in $G$ is denoted by $G_{\text {right }}$ (resp. $G_{l e f t}$ ).

\footnotetext{
${ }^{2}$ The notation $E_{n}$ has sense only if $n$ is a prime power.

${ }^{3}$ Sometimes this group is denoted by $Q D_{2^{k}}$ and called the quasidihedral group.

${ }^{4}$ This group is the $\operatorname{SmallGroup}(16,13)$ in GAP notation.
} 


\section{SCHUR RINGS AND CAYLEY SCHEMES}

In this section we recall some facts on association schemes and Cayley schemes that will be used throughout the paper to prove non-schurity of S-rings. More details can be found in [11].

Let $\Gamma$ be a transitive permutation group on a finite set $\Omega$. Denote by $\mathcal{R}$ the set of orbits in its coordinatetwise action on the Cartesian square $\Omega \times \Omega$. Then one can form a $\mathbb{Z}$-submodule $\mathcal{Z}=\mathcal{Z}(\Gamma, \Omega)$ in the $\operatorname{ring} \operatorname{Mat}_{\Omega}(\mathbb{Z})$ of all integer matrices the rows and columns of which are indexed by the elements of $\Omega$ :

$$
\mathcal{Z}=\operatorname{Span}\{A(R): R \in \mathcal{R}\}
$$

where $A(R)$ is the adjacency matrix of the binary relation $R \subset \Omega \times \Omega$. It is easily seen that $\mathcal{Z}$ is a matrix ring, the centralizer ring of the permutation group $\Gamma$. In the special case when $\Omega=G$ is a group and $G_{\text {right }} \leq \Gamma$ the injection

$$
\rho: G_{l e f t} \rightarrow \operatorname{Mat}_{\Omega}(\mathbb{Z}), g \mapsto P_{g},
$$

where $P_{g}$ is the permutation matrix associated with $g$, induces a ring isomorphism from $\mathcal{A}(\Gamma, G)$ onto $\mathcal{Z}(\Gamma, \Omega)$ [24, Theorem 28.8].

A partition $\mathcal{R}$ of the set $\Omega \times \Omega$ forms an association scheme $\mathcal{X}=(\Omega, \mathcal{R})$ if the module $\mathcal{Z}$ defined by (3) is a ring (the adjacency ring of $\mathcal{X}$ ) and

$$
1_{\Omega} \in \mathcal{R} \quad \text { and } \quad \mathcal{R}^{*}=\mathcal{R}
$$

where $1_{\Omega}$ is the diagonal of $\Omega \times \Omega$ and $\mathcal{R}^{*}$ consists of all relations $R^{*}$ obtained from $R \in \mathcal{R}$ by interchanging of coordinates 5 In the special case when $\mathcal{Z}=\mathcal{Z}(\Gamma, \Omega)$ for a permutation group $\Gamma$, the corresponding association scheme is called schurian and denoted by $\operatorname{Inv}(\Gamma)=\operatorname{Inv}(\Gamma, \Omega)$. For an arbitrary association scheme we define the automorphism group by

$$
\operatorname{Aut}(\mathcal{X})=\left\{\gamma \in \operatorname{Sym}(\Omega): R^{\gamma}=R, R \in \mathcal{R}\right\}
$$

where $R^{\gamma}=\left\{\left(\alpha^{\gamma}, \beta^{\gamma}\right):(\alpha, \beta) \in R\right\}$. The mappings Inv and Aut define a Galois correspondence between the association schemes with transitive automorphism groups and transitive permutation groups on $\Omega$ :

$$
\mathcal{X} \leq \operatorname{Inv}(\Gamma) \quad \Leftrightarrow \quad \Gamma \leq \operatorname{Aut}(\mathcal{X})
$$

where the partial order on the association schemes is induced by the natural partial order of their adjacency rings. The schurian schemes are closed with respect to this correspondence; in the other words, a scheme $\mathcal{X}$ is schurian if and only if $\mathcal{X}=\operatorname{Inv}(\operatorname{Aut}(\mathcal{X}))$. This observation gives a simple sufficient condition for a scheme to be non-schurian.

Lemma 2.1. A scheme $\mathcal{X}$ is non-schurian whenever $\mathcal{X} \neq \operatorname{Inv}(\operatorname{Aut}(\mathcal{X}))$.

A special class of association schemes $\mathcal{X}$ arises when $\Omega=G$ is a group and

$$
G_{\text {right }} \leq \operatorname{Aut}(\mathcal{X})
$$

In this case $\mathcal{X}$ is called a Cayley scheme over the group $G$; any relation $R \in \mathcal{R}$ is the arc set of the Cayley graph on $G$ associated with a set $X=\{g \in G:(g, e) \in R\}$. The set of all such $X$ 's forms a partition $\mathcal{S}$ of the group $G$ for which the $\mathbb{Z}$-module (1)

\footnotetext{
${ }^{5}$ This definition of an association scheme is obviously equivalent to the standard one.
} 
is an S-ring; it is denoted by $\mathcal{A}(\mathcal{X})$. Conversely, by the definition of mapping (4) for any set $X \subset G$ the matrix

$$
\rho(\underline{X})=\sum_{g \in X} P_{g}
$$

is a $\{0,1\}$-matrix in $\operatorname{Mat}_{\Omega}(\mathbb{Z})$. Therefore it is the adjacency matrix of a binary relation $R \subset G \times G$ which is invariant with respect to $G_{\text {right }}$. One can also see that all these relations form a partition $\mathcal{R}$ of the set $G \times G$ for which conditions (5) are satisfied. Thus the pair $\mathcal{X}(\mathcal{A})=(G, \mathcal{R})$ is a Cayley scheme over the group $G$.

Theorem 2.2. The correspondence $\mathcal{A} \mapsto \mathcal{X}(\mathcal{A}), \mathcal{X} \mapsto \mathcal{A}(\mathcal{X})$ induces a bijection between the $S$-rings and Cayley schemes over the group $G$ that preserves the natural partial orders on these sets. Moreover, under this correspondence the $S$-ring $\mathcal{A}$ is schurian if and only if so is the Cayley scheme $\mathcal{X}(\mathcal{A})$.

We conclude the section by two results giving necessary conditions for a group $G$ to be Schur. Below given a group $H \leq G$ we denote by $\Gamma(H, G)$ the (transitive) permutation group which is induced by the action of the group $G$ on the set of right $H$-cosets by the right multiplications. In the proof of the following lemma we will freely use well-known properties of the wreath product of association schemes; all of them can be found, e.g. in [8].

Lemma 2.3. Let $G$ be a Schur group, $H \leq G$ and $\Gamma=\Gamma(H, G)$. Then any fusion 6 of the scheme $\operatorname{Inv}(\Gamma)$ is schurian.

Proof. It is easily seen that the set of right $H$-cosets forms an imprimitivity system of the group $G_{\text {right }}$. Therefore without loss of generality we can assume that it is a subgroup of the wreath product $\Delta=H_{\text {right }} 2 \Gamma$ in imprimitive action. This implies that

$$
G_{\text {right }} \leq \Delta \leq \operatorname{Aut}(\operatorname{Inv}(\Delta))=\operatorname{Aut}\left(\mathcal{X}_{H} \prec \mathcal{X}_{\Gamma}\right)
$$

where $\mathcal{X}_{H}=\operatorname{Inv}\left(H_{\text {right }}\right)$ and $\mathcal{X}_{\Gamma}=\operatorname{Inv}(\Gamma)$. It follows that $\mathcal{X}_{H} \prec \mathcal{X}_{\Gamma}$ is a Cayley scheme over the group $G$. Now, suppose on the contrary that there exists a nonschurian fusion $\mathcal{Y}$ of the scheme $\mathcal{X}_{\Gamma}$. Then

$$
\mathcal{X}_{H} \prec \mathcal{Y} \leq \mathcal{X}_{H} \prec \mathcal{X}_{\Gamma}
$$

and hence $\mathcal{X}_{H} \mathcal{Y Y}$ is also a Cayley scheme over $G$. Moreover, this scheme is schurian if and only if so is the scheme $\mathcal{Y}$ (see e.g. [8]). By Theorem 2.2 this implies that the S-ring $\mathcal{A}\left(\mathcal{X}_{H} \imath \mathcal{Y}\right)$ is not schurian. Therefore the group $G$ is not Schur. Contradiction.

When the group $H$ in Lemma 2.3 is normal in $G$, the scheme $\operatorname{Inv}(\Gamma)$ is obviously a Cayley scheme over the group $G / H$. Therefore our result implies that the quotient of a Schur group is also Schur. This provides a proof of one part of the following theorem proved in 20 .

Theorem 2.4. Any section of a Schur group is a Schur group.

\footnotetext{
${ }^{6}$ A scheme $\mathcal{Y}$ is called a fusion of a scheme $\mathcal{X}$, if $\mathcal{Y} \leq \mathcal{X}$
} 
TABLE 1. Non-schurian schemes for small groups

\begin{tabular}{|c|c|c|c|}
\hline$G$ & $\mathcal{X}$ & $\operatorname{rk}(\mathcal{X})$ & $\operatorname{rk}(\Gamma)$ \\
\hline$[16,3]$ & {$[16,59]$} & 6 & 7 \\
\hline$[16,6]$ & {$[16,6]$} & 3 & 4 \\
\hline$[16,9]$ & {$[16,59]$} & 6 & 7 \\
\hline$[16,12]$ & {$[16,59]$} & 6 & 7 \\
\hline$[18,4]$ & {$[18,41]$} & 6 & 8 \\
\hline$[24,3]$ & {$[24,308]$} & 8 & 9 \\
\hline$[24,5]$ & {$[24,299]$} & 8 & 14 \\
\hline$[24,8]$ & {$[24,299]$} & 8 & 14 \\
\hline$[24,11]$ & {$[24,308]$} & 8 & 9 \\
\hline$[24,13]$ & {$[24,106]$} & 6 & 12 \\
\hline$[27,3]$ & {$[27,382]$} & 4 & 6 \\
\hline
\end{tabular}

\begin{tabular}{|c|c|c|c|}
\hline$G$ & $\mathcal{X}$ & $\operatorname{rk}(\mathcal{X})$ & $\operatorname{rk}(\Gamma)$ \\
\hline$[16,4]$ & {$[16,94]$} & 7 & 10 \\
\hline$[16,8]$ & {$[16,6]$} & 3 & 4 \\
\hline$[16,11]$ & {$[16,6]$} & 3 & 4 \\
\hline$[18,3]$ & {$[18,41]$} & 6 & 8 \\
\hline$[24,1]$ & {$[24,191]$} & 7 & 24 \\
\hline$[24,4]$ & {$[24,304]$} & 8 & 14 \\
\hline$[24,7]$ & {$[24,304]$} & 8 & 14 \\
\hline$[24,10]$ & {$[24,304]$} & 8 & 14 \\
\hline$[24,12]$ & {$[24,17]$} & 4 & 6 \\
\hline$[24,14]$ & {$[24,299]$} & 8 & 14 \\
\hline$[27,4]$ & {$[27,382]$} & 4 & 6 \\
\hline
\end{tabular}

\section{Abelian and small nOn-Abelian Schur groups}

All S-rings over any group of order $n \leq 31$ were enumerated by computer in [12]; the same result for $n \leq 47$ was later announced in [19]. As a consequence of these results one can get Lemma 3.1 below. An independent proof of this lemma we give here, is obtained by using the enumeration of all association schemes (and hence the Cayley schemes) of small order in [15].

Lemma 3.1. A nonabelian group of order $\leq 27$ is not Schur unless it is a dihedral group or is isomorphic to $Q_{8}, A_{4}, C_{3}: C_{4}$, or $G_{16}$.

Proof. By means of computer system GAP [13] one can find that there are exactly 22 nonabelian groups that are neither dihedral nor isomorphic to $Q_{8}, A_{4}$, $C_{3}: C_{4}$, or $G_{16}$. All that groups are listed in the first column of the Table 1 for instance, the third row of the left-hand side correspond to the group $Q_{16}$, the SmallGroup $(16,9)$ in GAP.

For each group $G$ in this list we choose an association scheme $\mathcal{X}=(\Omega, \mathcal{R})$ belonging to the Hanaki-Miamoto database of small association schemes [15] where $\Omega=\{1, \ldots, n\}$ with $n=|G|$. The second and third columns of the table contain the index of the scheme $\mathcal{X}$ in the database and the number $\operatorname{rk}(\mathcal{X})=|\mathcal{R}|$ respectively.

By means of the Hanaki GAP-package for association schemes [16] one can find the group $\Gamma=\operatorname{Aut}(\mathcal{X})$; the number $\operatorname{rk}(\Gamma)=\operatorname{rk}(\operatorname{Inv}(\Gamma))$ is given in the fourth column of the Table 1 Inspecting the table shows that $\operatorname{rk}(\mathcal{X}) \neq \operatorname{rk}(\Gamma)$, and hence

$$
\mathcal{X} \neq \operatorname{Inv}(\operatorname{Aut}(\mathcal{X})) .
$$

By Lemma 2.1 this implies that the scheme $\mathcal{X}$ is non-schurian. On the other hand, a straightforward computation in GAP shows that the group $\Gamma$ contains a regular subgroup isomorphic to $G$. Therefore the scheme $\mathcal{X}$ is isomorphic to a Cayley scheme over this group. Thus the group $G$ is not Schur by Theorem 2.2.

The next two statements contain almost all known information on large Schur groups. The first of them was proved in 20.

Theorem 3.2. A p-group with $p \geq 5$ is Schur if and only if it is cyclic. 
The following theorem is a consequence of the results obtained in 9 for the cyclic case and in 10, for the abelian non-cyclic case.

Theorem 3.3. Any abelian Schur group is contained in one of the following families:

(1) $C_{p^{k}}, C_{p q^{k}}, C_{2 p q^{k}}, C_{p q r}, C_{2 p q r}$,

(2) $E_{p^{k}}$ where either $p=2$ and $k \leq 5$, or $p=3$ and $k \leq 3$,

(3) $C_{p} \times C_{p^{k}}$ where $p=2$ or $p=3$,

(4) $C_{2 p} \times C_{2^{k}}, E_{4} \times C_{p^{k}}, E_{4} \times C_{p q}, E_{16} \times C_{p}$ where $p \neq 2$,

(5) $C_{6} \times C_{3^{k}}, E_{9} \times C_{2 q}, E_{9} \times C_{p}$ where $p \neq 2$

with $p, q, r$ being distinct primes and $k \geq 0$ an integer.

Remark 3.4. From [9] it follows that all the groups in cases (1) and (2) are Schur.

Corollary 3.5. Let $G$ be an abelian Schur group of order $n$ and $\Omega(n)$ the total number of prime factors of the integer $n$. Then

(1) $\pi(G) \leq 4$ with equality only if $2 \in \pi(G)$ and $\Omega(n)=4$,

(2) if $n$ is odd, then $\pi(G) \leq 3$ with equality only if $\Omega(n)=3$.

In the following two lemmas below we prove that several small groups are not Schur. In all cases we find a transitive permutation group $\Gamma \leq \operatorname{Sym}(\Omega)$ and an association scheme $\mathcal{X}=(\Omega, \mathcal{R})$ such that

$$
\mathcal{X} \neq \operatorname{Inv}(\Gamma) \text { and } \operatorname{Aut}(\mathcal{X})=\Gamma
$$

By Lemma 2.1 this scheme is non-schurian. To define the scheme $\mathcal{X}$ we will specify the nonreflexive orbits $R_{1}, \ldots, R_{k}$ in the coordinatewise action of the group $\Gamma$ on the set $\Omega \times \Omega$. Then we choose a nontrivial partition $\Pi$ of the set $\{1, \ldots, k\}$, and define a nonreflexive element of $\mathcal{R}$ to be the union of all $R_{i}$ 's belonging to a class of $\Pi$. In each case one can easily check by means of GAP that (a) $\mathcal{X}$ is an association scheme and (b) relations (6) hold.

Lemma 3.6. The Frobenius groups $E_{8}: C_{7}$ and $E_{16}: C_{3}$ are not Schur.

Proof. Let $G$ be one of the groups in the lemma statement. Suppose we are given a permutation group $\Gamma$ and scheme $\mathcal{X}$ for which relations (6) hold with $\Omega=G$. If, in addition,

$$
G_{\text {right }} \leq \Gamma,
$$

then $\mathcal{X}$ is isomorphic to a non-schurian Cayley scheme over $G$. However, then the S-ring $\mathcal{A}(\mathcal{X})$ is also non-schurian by Theorem 2.2. Thus the group $G$ can not be Schur. To complete the proof let us construct the group $\Gamma$ and scheme $\mathcal{X}$ in each case.

Let $G=E_{8}: C_{7}=\operatorname{SmallGroup}(56,11)$. The group $\operatorname{SmallGroup}(672,1257)$ is solvable and can be written as a product of two subgroups that are isomorphic to $G$ and $A_{4}$ respectively. This decomposition is not unique and the rank of the permutation representation on the right cosets of $A_{4}$ can be 8,12 or 20 . In the first case, there are three conjugacy classes of subgroups isomorhic to $A_{4}$, that produce such a representation. Denote by $\Gamma$ the permutation group that corresponds to one of them. Then obviously $\Gamma \geq G_{\text {right }}$ and the above defined number $k$ is equal to 7 . Moreover, the indices of the relations $R_{i}$ 's can be chosen so that

$$
n_{1}=1, n_{2}=n_{3}=3, n_{4}=\cdots=n_{7}=12
$$


where $n_{i}=\left|R_{i}\right| /|\Omega|, R_{2}$ but not $R_{3}$ is the union of 7 's complete graphs on 4 vertices, and $\left(R_{4}\right)^{*}=R_{5}$ and $\left(R_{6}\right)^{*}=R_{7}$. Then we are done with the partition $\Pi=\{\{1,3\},\{2\},\{4,5\},\{6,7\}\}$.

Let $G=E_{16}: C_{3}=\operatorname{SmallGroup}(48,50)$. The group SmallGroup $(1152,154768)$ is solvable and is isomorpic to a semidirect product of $G$ by $\mathrm{SL}_{2}(3)$. Moreover, there is a unique (up to conjugacy) subgroup isomorphic to $\mathrm{SL}_{2}(3)$ such that the permutation representation on the right cosets of this group has rank 9 and contains no a suborbit of size 3 . Denote the corresponding permutation group by $\Gamma_{1}$. Then $N_{\text {Sym }(\Omega)}\left(\Gamma_{1}\right) \simeq E_{4}$. Over three proper subgroups of $N_{\text {Sym }(\Omega)}\left(\Gamma_{1}\right)$ that properly contain $\Gamma_{1}$ there are two of rank 6 . Denote by $\Gamma$ any of them. Then obviously $\Gamma \geq G_{\text {right }}$ and the above defined number $k$ is equal to 5 . Moreover, the indices of the relations $R_{i}$ 's can be chosen so that

$$
n_{1}=1, n_{2}=2, n_{3}=12, n_{4}=n_{5}=16 .
$$

Then we are done with the partition $\Pi=\{\{1\},\{2,3\},\{4\},\{5\}\}$.

Lemma 3.7. The Frobenius group $E_{16}: C_{5}$ and the group $A_{5}$ are not Schur.

Proof. For each group $G$ from the lemma statement we choose a subgroup $H$. By Lemma 2.3 it suffices to find a non-schurian fusion of the scheme $\mathcal{Y}=\operatorname{Inv}(\Delta, \Omega)$ where $\Delta=\Gamma(H, G)$ and $\Omega$ is the set of all right $H$-cosets. For this we will explicitly define a permutation group $\Gamma$ such that

$$
\operatorname{Aut}(\mathcal{Y}) \leq \Gamma,
$$

and association scheme $\mathcal{X}$ for which relations (6) hold. Then as above $\mathcal{X}$ is a required non-schurian fusion of $\mathcal{Y}$ and we are done.

Let $G=E_{16}: C_{5}=$ SmallGroup $(80,49)$. Set $H$ to be a subgroup of $G$ that is generated by an involution (all these subgroups belong to the same orbit of $\operatorname{Aut}(G)$. Then the factor group $N_{\mathrm{Sym}(\Omega)}(\Delta) / \Delta$ has a unique normal subgroup isomorphic to $C_{4} \times C_{2}$. Denote by $\Gamma$ the permutation group such that

$$
\Delta \leq \Gamma \leq N_{\operatorname{Sym}(\Omega)}(\Delta)
$$

and the factor group $\Gamma / \Delta$ coincides with that normal subgroup. Then the above defined number $k$ is equal to 9 and the indices of the relations $R_{i}$ 's can be chosen so that

$$
n_{1}=n_{2}=n_{3}=1, n_{4}=n_{5}=2, n_{6}=\cdots=n_{9}=8,
$$

and we are done with the partition $\Pi$ the unique non-singleton class of which is equal to $\{4,5\}$.

Let $G=A_{5}=$ SmallGroup $(60,5)$. Set $H$ to be the subgroup of $G$ that is generated by the involution $(1,2)(3,4)$. Denote by $\Gamma$ the permutation group $N_{\operatorname{Sym}(\Omega)}(\Delta)$. Then the above defined number $k$ is equal to 6 and the indices of the relations $R_{i}$ 's can be chosen so that

$$
n_{1}=1, n_{2}=n_{3}=n_{4}=4, n_{5}=n_{6}=8,
$$

and the relations $R_{2}$ and $R_{3}$ (not $R_{4}$ ) form simple connected graphs with the vertex set $\Omega$. In this case we are done with the partition $\Pi$ the unique non-singleton class of which is equal to $\{2,3\}$.

We complete the section by an auxiliary lemma which is a consequence of the above results, and will be used in Section 7 . 
TABLE 2. Reasons for groups from Lemma 3.8 to be non-schurian

\begin{tabular}{|c|c|c|c|c|}
\hline$k$ & $p$ & $c$ & $K$ & \\
\hline 3 & 3 & 2 & $A_{4} \times C_{2}$ & Lemma $[3.1$ \\
\hline 3 & 7 & 1 & $E_{8}: C_{7}$ & Lemma $[3.6$ \\
\hline 4 & 3 & 1 & $E_{16}: C_{3}$ & Lemma $[3.6$ \\
\hline 4 & 3 & 4 & $A_{4} \times C_{2}$ & Lemma $[3.1$ \\
\hline 4 & 5 & 1 & $E_{16}: C_{5}$ & Lemma $[3.7$ \\
\hline 4 & 7 & 2 & $E_{8}: C_{7}$ & Lemma $[3.6$ \\
\hline 5 & 3 & 8 & $A_{4} \times C_{2}$ & Lemma 3.1 \\
\hline 5 & 3 & 2 & $E_{16}: C_{3}$ & Lemma $[3.6$ \\
\hline 5 & 5 & 2 & $E_{16}: C_{5}$ & Lemma $[3.7$ \\
\hline 5 & 7 & 4 & $E_{8}: C_{7}$ & Lemma $[3.6$ \\
\hline 5 & 31 & 1 & $E_{32}: C_{31}$ & \\
\hline
\end{tabular}

Lemma 3.8. Let $G$ be a Schur group and $P$ a Sylow 2-subgroup of $G$. Suppose that $P$ is proper, normal, elementary abelian, and $C_{G}(P)=P$. Then $G \simeq A_{4}$ or $G \simeq E_{32}: C_{31}$.

Proof. According to the Schur-Zassenhaus Theorem [14, p.221], the group $P$ being a normal Sylow subgroup has a complement in $G$; in other words, there exists a group $H \leq G$ of order $|G: P|$ such that $G=P H$. In particular, $H$ is of odd order. Taking into account that $C_{G}(P)=P$ and $P \simeq E_{2^{k}}$ for some integer $k \geq 1$, without loss of generality we can assume that

$$
H \leq \mathrm{GL}_{k}(2)
$$

Moreover, by statement (2) of Theorem 3.3 we have $2 \leq k \leq 5$ because $P$ is proper and $G$ is Schur. When $k=2$, then obviously $G L_{2}(2) \simeq S_{3}$ and $|H|=3$. Therefore, $G \simeq A_{4}$ and and we are done. In the remaining cases take a nonidentity element $x \in H$ of prime order $p$. Then by (7) we have

$$
p= \begin{cases}3 \text { or } 7, & \text { if } k=3, \\ 3,5, \text { or } 7, & \text { if } k=4, \\ 3,5,7 \text { or } 31, & \text { if } k=5 .\end{cases}
$$

In the Table 2 we list all possible triples $(k, p, c)$ with $k=3,4,5$ and $c=\left|C_{P}(x)\right|$. For each such triple the fourth column of this table contains a group $K$ which is isomorphic to a subgroup of $P\langle x\rangle$. All these groups except for one in the last row, are non-Schur and the reason for this is given in the fifth column. To complete the proof it suffices to note that in the last row $K=H$ is a Sylow 31-subgroup of $\mathrm{GL}_{5}(2)$ that is cyclic.

\section{Non-ABelian Schur $p$-GRoups}

In this section we are interested in non-abelian nilpotent Schur groups. Since any nilpotent group is the direct product of $p$-groups and for $p \geq 5$ there are no non-cyclic Schur $p$-groups, it is quite natural to begin with studying 2 - and 3 -groups. 
We recall that the rank of an abstract finite group $G$ is the least positive integer $r=r(G)$ such that every subgroup of $G$ is generated by $r$ elements. When $G$ is a $p$-group for a prime $p$, then by the Burnside theorem on a basis of a $p$-group $r(G)=r$ if and only if $E_{p^{r}} \prec G$ and $E_{p^{r+1}} \nprec G$.

Lemma 4.1. A non-abelian Schur p-group of rank $r \geq 3$ is isomorphic to the group $G_{16}$.

Proof. Let $G$ be a non-abelian Schur $p$-group of rank $r \geq 3$. Then it has an elementary abelian section $U / L$ of order $p^{r}$ such that $U \neq G$ or $L \neq 1$. If $L \neq 1$, then $U$ contains a normal subgroup $L^{\prime}<L$ with $\left|L / L^{\prime}\right|=p$, and we set $H=U / L^{\prime}$. If $L=1$, then the normalizer of $U$ in $G$ contains a subgroup $U^{\prime}$ with $\left|U / U^{\prime}\right|=p$, and we set $H=U^{\prime} / L$. Thus in any case $G$ has a section $H$ such that $|H|=p^{r+1}$ and $r(H)=r$. By Theorem 2.4 the group $H$ is Schur. However, by Theorem 3.3 any abelian Schur group of rank at least 3 is elementary abelian. Thus $H$ is not abelian (otherwise $r(H)=r+1$ ). Finally, $p=2$ or $p=3$ by Theorem 3.2 Let us consider these two cases separately.

Case $p=3$. Without loss of generality we can assume that $H$ is a nonabelian group of order 81 and rank 3. By means of GAP one can find that SmallGroup $(81,6)=C_{27}: C_{3}$ is a unique non-abelian group of order 81 that has no section isomorphic to $C_{9}: C_{3}$ or $E_{9}: C_{3}$. However, by Lemma 3.1 none of latter groups is Schur. Since the group $H$ is Schur, Theorem 2.4 implies that $H \simeq C_{27}: C_{3}$. But then $r(H)=2$ which contradicts the choice of $H$.

Case $p=2$. Without loss of generality we can assume that $H$ is a non-abelian group of order 64 and rank 5. However, there are exactly nine such groups and each of them has a subgroup isomorphic to $C_{4} \times E_{4}$. By Theorem 3.3, this group, and hence $H$ is not Schur. Contradiction. Thus $r=3$ or $r=4$.

Let $r=4$. In this case $H$ is a non-abelian group of order 32 and rank 4 . However, there are exactly seven such groups and each of them has a section isomorphic to $C_{4} \times E_{4}, C_{2} \times D_{8}$ or $C_{2} \times Q_{8}$. All of these groups are not Schur: the former one by Theorem 3.3 whereas the latter two by Lemma 3.1. It follows that the group $H$ is not Schur. Contradiction.

Let $r=3$. In this case $H$ is a non-abelian group of order 16 and rank 3. However, there are exactly four such groups that have a section isomorphic to $E_{8}$, namely,

$$
\left(C_{4} \times C_{2}\right): C_{2}, \quad C_{2} \times D_{8}, \quad C_{2} \times Q_{8}, \quad G_{16} .
$$

The first three groups are not Schur by Lemma 3.1 whereas the fourth one is Schur: this can be checked by enumeration of all S-rings over $G_{16}$. To complete the proof we will verify that any group $K$ of order 32 such that $G_{16} \prec K$, is not Schur. For this one can find that $K$ is isomorphic to one of 17 groups of order 32 , and each of these groups contains at least one subgroup isomorphic to one of the groups below:

$$
C_{4} \times C_{4}, \quad C_{4} \times E_{4}, \quad C_{8}: C_{2}, \quad S D_{16}, \quad C_{2} \times D_{8}, \quad C_{2} \times Q_{8} .
$$

All these groups are not Schur: the first three by Theorem 3.3 whereas the other four by Lemma 3.1. Thus the group $K$ is not Schur.

Theorem 4.2. A non-abelian $p$-group is not Schur unless $p=2$ or $p=3$, and it is isomorphic to one of the groups below:

(1) $Q_{8}, G_{16}, M_{2^{k}}, k>5$, or $D_{2^{k}}, k>2$, if $p=2$; 
(2) $M_{3^{k}}, k>3$, if $p=3$.

Morover, the groups $Q_{8}, G_{16}, D_{2^{k}}, 2<k<6$, are Schur.

Proof. Let $G$ be a non-abelian $p$-group. Then $p=2$ or $p=3$ by Theorem 3.2 Moreover, by Lemma 4.1 we can assume that $r(G)=2$. Denote by $\Phi=\Phi(G)$ the Frattini subgroup of the group $G$. Then $G / \Phi \simeq E_{p^{r}}$ for an integer $r \geq 1$, and $r=1$ if and only if the group $G$ is cyclic. By the above assumption this implies that $r=2$. We claim that the group $\Phi$ is cyclic. Suppose on the contrary that it is not true. Then again by the assumption we have $\Phi / \Phi_{2} \simeq E_{p^{2}}$ where $\Phi_{2}=\Phi(\Phi(G))$. Therefore $G$ contains a section $H=G / \Phi_{2}$ such that

$$
E_{p^{2}} \triangleleft H \quad \text { and } \quad H / E_{p^{2}} \simeq E_{p^{2}},
$$

in particular, $|H|=p^{4}$ and $r(H)=2$. Moreover, the group $H$ is not abelian, because otherwise $H \simeq C_{p^{2}} \times C_{p^{2}}$, and hence $H$ is not Schur by Theorem 3.3 Next, there are exactly five (resp. nine) non-abelian 2-groups (res. 3 -groups) $H$ satisfying (8). When $p=2$ four groups are not Schur by Lemma 3.1 whereas the fifth group is $G_{16}$ which is impossible because $r\left(G_{16}\right)=3$. When $p=3$ each of that nine groups contains a section isomorphic to either $E_{9}: C_{3}$ or $C_{9}: C_{3}$ which are not Schur also by Lemma 3.1, Thus the group $H$ is not Schur. The claim is proved.

From the above claim it follows that $G / \Phi^{2}$ is a group of order $p^{3}$ that has a quotient isomorphic to $E_{p^{2}}$. Since $r=2$, it is not isomorphic to $E_{p^{3}}$. Moreover, by Lemma 3.1 it is also not isomorphic to a non-abelian group of order 27 and exponent 3. Therefore the exponent of $G / \Phi^{2}$ equals $p^{2}$. Thus the $p$-group $G$ contains a cyclic subgroup of index $p$. So by the Burnside theorem [2, Theorem 1.2] one of the following statements hold:

(1) $p=3$ and $G \simeq M_{3^{k}}$ where $k \geq 3$,

(2) $p=2$ and $G$ is isomorphic to one of the groups $M_{2^{k}}, Q_{2^{k}}, S D_{2^{k}}$ for $k \geq 4$,

(3) $p=2$ and $G \simeq D_{2^{k}}$ for $k \geq 3$.

However, both the group $Q_{2^{k}}$ and the group $S D_{2^{k}}$ contains a subgroup isomorphic to $Q_{2^{k-1}}$ for $k \geq 5$. On the other hand, the groups $Q_{16}$ and $S D_{16}$ are not Schur by Lemma 3.1. Thus $G$ can not be isomorphic to $S D_{2^{k}}$, and is isomorphic to $Q_{2^{k}}$ only for $k=3$. This completes the proof of the first statement. The second follows by a computer enumeration of all S-rings over small groups.

Recall that a finite nilpotent group is a direct product of its Sylow subgroup. Therefore, the next assertion is a direct consequence of Theorem 3.3 on a structure of abelian Schur groups and the last theorem.

Corollary 4.3. Let $G$ be a nilpotent Schur group. Then there is at most one prime $p$ such that a Sylow p-subgroup of $G$ is non-cyclic, and $p$ is equal to 2 or 3. Moreover, $G$ is a metabelian group with $r(G) \leqslant 5$ and $|\pi(G)| \leqslant 4$, and if $|\pi(G)|=4$, then $G$ is a cyclic group of even order, which is the product of four primes.

\section{Case of dihedral group}

In this section we are interested in non-Schur dihedral groups. Let us begin with a construction producing S-rings of rank 4; in fact, this construction can be considered as a special case of one that used in [3, Theorem 1.6.1] to establish a 
well-known relationship between symmetric block designs and bipartite distance regular graphs of diameter 3.

Let $G$ be the generalized dihedral group associated with abelian group $H$, i.e $G=\langle H, g\rangle$ with $h^{g}=h^{-1}$ for all $h \in H$. Suppose that $D \subset H$ is a difference set in $H$; by definition this means that

$$
\left(\underline{D} \cdot \underline{D}^{-1}\right) \circ \underline{A}=\lambda \underline{A}
$$

for a positive integer $\lambda$ where $\circ$ is the componentwise multiplication in the group ring $\mathbb{Z} H$ and $A$ is the set of nonidentity elements of $H$. Denote by $\mathcal{S}$ the partition of the elements of $G$ into four classes:

$$
\{e\}, \quad A, \quad X=D g, \quad Y=(H \backslash D) g
$$

where $e=e_{G}$. Then obviously $A^{-1}=A$. Taking into account that $g^{2}=e$, we obtain that $X^{-1}=g^{-1} D^{-1}=D g=X$ and similarly $Y^{-1}=Y$. Thus condition (2) is satisfied for the partition $\mathcal{S}$. Furthermore, it is easily seen that

$$
\begin{aligned}
& \underline{A} \cdot \underline{A}=(n-1) e+(n-2) \underline{A} \\
& \underline{A} \cdot \underline{X}=(k-1) \underline{X}+k \underline{Y} \\
& \underline{A} \cdot \underline{Y}=(n-k) \underline{X}+(n-k-1) \underline{Y}
\end{aligned}
$$

where $n=|H|$ and $k=|D|$. From (9) it also follows that

$$
\underline{X} \cdot \underline{Y}=\underline{D g} \cdot \underline{H g \backslash D g}=\underline{D} \cdot \underline{H}-\underline{D} \cdot \underline{D}^{-1}=k e+(k+\lambda) \underline{A} .
$$

Finally, $\underline{U} \cdot \underline{V}=(\underline{V} \cdot \underline{U})^{-1}=\underline{V} \cdot \underline{U}$ for all $U, V \in \mathcal{S}$. Thus we obtain the following statement.

Lemma 5.1. The $\mathbb{Z} G$-module $\mathcal{A}=\mathcal{A}(D, H)=\operatorname{Span}\{\underline{Z}: Z \in \mathcal{S}\}$ is an $S$-ring over the group $G$.

Starting with the difference set $D$ one can construct in a standard way a symmetric 2-design $\mathcal{B}=\operatorname{dev}(D)$ the points, blocks and flags of which are respectively the elements of the group $H$, the elements of coset $H g$, and the pairs $(x, y)$ with $y x^{-1} \in D g$ The set of flags is obviously invariant with respect to the setwise stabilizer of the set $H$ in the group $G_{\text {right }}$. Therefore this stabilizer forms an automorphism group of the design $\mathcal{B}$ that acts regularly both on the points and on the blocks. The design is 2-transitive (resp. flag-transitive, antiflag-transitive) if the group $\operatorname{Aut}(\mathcal{B})$ acts 2 -transitively on the points (resp. acts transitively on the flags, on the anti-flags).

Lemma 5.2. The $S$-ring $\mathcal{A}$ is schurian if and only if the design $\mathcal{B}$ is 2-transitive, flag-transitive and antiflag-transitive.

Proof. Suppose that the S-ring $\mathcal{A}$ is schurian. Then there exists a permutation group $\Gamma \leq \operatorname{Sym}(G)$ such that

$$
G_{\text {right }} \leq \Gamma \quad \text { and } \quad A, X, Y \in \operatorname{Orb}\left(\Gamma_{e}\right)
$$

where $\Gamma_{e}$ is the stabilizer of $e$ in $\Gamma$. The setwise stabilizer $\Delta$ of the set $H$ in $\Gamma$ acts in a natural way on both $H$ and $H g$. Moreover, it is easily seen that

$$
H_{\text {right }} \leq \Delta^{H} \text { and } \quad A \in \operatorname{Orb}\left(\left(\Delta^{H}\right)_{e}\right) .
$$

\footnotetext{
${ }^{7}$ The defining property of a 2-design is that any pair of distinct points is incident to the same number of blocks; the design is symmetric if the number of points is equal to the number of blocks.
} 
Therefore the action of the group $\Delta$ on the points of $\mathcal{B}$ is 2-transitive. Moreover, taking into account that $X$ and $Y$ are orbits of $\Gamma_{e}$, and hence of $\Delta_{e}$, we conclude that the sets

$$
\bigcup_{\delta \in \Delta}(e, X)^{\delta} \text { and } \bigcup_{\delta \in \Delta}(e, Y)^{\delta}
$$

coincide respectively with the sets of flags and anti-flags of the design $\mathcal{B}$. Therefore $\Delta$ is an automorphism group of this design that acts transitively on its flags and anti-flags. Thus the design $\mathcal{B}$ is 2 -transitive, flag-transitive and antiflag-transitive. The converse statement is proved in a similar way.

Given a prime power $q=4 a+3, a \geq 1$, there is the Paley difference set $D$ in the additive group $H$ of a finite field with $q$ elements, that consists of $k=(q-1) / 2$ non-zero squares. In this case for all $k \geq 5$ we obviously have

$$
1+\sqrt{k}>(q-1) / k=2 .
$$

Therefore by [17, Theorem 8.3] the $\operatorname{design} \operatorname{dev}(D)$ is 2-transitive only if $q=7$ or $q=11$. So by Lemma 5.2 the S-ring $\mathcal{A}(D, H)$ is not schurian for $q \geq 13$. Since the group $G=D_{2 q}$ is Schur for $q \leq 11$, we obtain the following statement.

Corollary 5.3. Let $p \equiv 3(\bmod 4)$ be a prime. Then a dihedral group of order $2 p$ is a Schur group if and only if $p \leq 11$.

To formulate the next result we recall that a difference set is nontrivial if neither it nor its complement is a singleton. Two difference sets $D$ and $D^{\prime}$ are isomorphic if so are the corresponding designs $\operatorname{dev}(D)$ and $\operatorname{dev}\left(D^{\prime}\right)$. Given a prime power $q$ and integer $d \geq 2$ denote by $D=S_{q, d}$ the Singer difference set: the parameters of it are as follows

$$
(n, k, \lambda)=\left(\left(q^{d+1}-1\right) /(q-1),\left(q^{d}-1\right) /(q-1),\left(q^{d-1}-1\right) /(q-1)\right),
$$

and the points and blocks of the design $\operatorname{dev}(D)$ are the lines and hyperplanes of a linear space of dimension $d$ over a finite field with $q$ elements.

Corollary 5.4. Let $G$ be the generalized dihedral group associated with abelian group $H$ in which there exists a nontrivial difference set. Then $G$ is a Schur group only if this difference set is isomorphic to a Singer difference set. In particular, in this case $|H|=\left(q^{d+1}-1\right) /(q-1)$ for a prime power $q$ and integer $d \geq 2$.

Proof. Let $D$ be the difference set from the corollary hypothesis. Suppose that the group $G$ is Schur. Then the S-ring $\mathcal{A}(D, H)$ must be schurian. So by Lemma 5.2 the design $\operatorname{dev}(D)$ is 2-transitive and antiflag-transitive. According to [7, Statement 35a, p.91] this implies that this design is isomorphic to the $\operatorname{design} \operatorname{dev}(S)$ where $S=S_{q, d}$ for some $d$ and $q$. Thus the difference set $D$ is isomorphic to a Singer difference set and we are done.

A lot of information on difference sets with parameters (10) is contained in [4. In particular, one can find cyclic groups of order $n=\left(q^{d+1}-1\right) /(q-1)$ where $q$ is a prime power and $d \geq 2$, each of which admits a difference set with parameters (10) that is not isomorphic to a Singer difference set. By Corollary 5.4 this implies that in all these cases the group $D_{2 n}$ is not Schur. 


\section{Proof of Theorem 1.2}

Due to Remark 3.4, it suffices to verify that any non-abelian simple group is not Schur. However, such a group always contains a minimal simple group, i.e a nonabelian simple group of composite order all of whose proper subgroups are solvable. So by Theorem 2.4 only we need is to check that any minimal non-abelian simple group $G$ is not Schur. By the Thompson Theorem [22, Corollary 1] the group $G$ is isomorphic to one of the following groups:

(T1) $\operatorname{PSL}_{2}(p)$ where $p$ is a prime, $p>3$, and $p \equiv \pm 2(\bmod 5)$,

(T2) $\mathrm{PSL}_{2}\left(3^{p}\right)$ where $p$ is an odd prime,

(T3) $\mathrm{PSL}_{2}\left(2^{p}\right)$ where $p$ is a prime,

(T4) $\mathrm{Sz}\left(2^{p}\right)$ where $p$ is an odd prime,

(T5) $\mathrm{PSL}_{3}(3)$.

In each of cases (T3)-(T5) the group $G$ contains a subgroup isomorphic to a nonSchur group $H$ : the second and third columns of Table 3 below indicate the group $H$ and the reason of why it is non-Schur (the existence of such a subgroup is clear, see, e. g., [5, 23]). Thus in all these cases the group $G$ is not schur by Theorem 2.4.

TABLE 3. Non-schurian subgroups of some simple groups

\begin{tabular}{|l|l|l|}
\hline$G$ & $H$ & non-Schur \\
\hline $\mathrm{PSL}_{2}(4)$ & $A_{5}$ & Lemma 3.7 \\
\hline $\mathrm{PSL}_{2}(8), \mathrm{Sz}(8)$ & $E_{8}: C_{7}$ & Lemma [3.6 \\
\hline $\mathrm{PSL}_{2}(32), \mathrm{Sz}(32)$ & $D_{62}$ & Corollary [5.3 \\
\hline $\mathrm{PSL}_{2}\left(2^{p}\right), \mathrm{Sz}\left(2^{p}\right), p>5$ & $E_{2^{p}}$ & Theorem 4.2 \\
\hline $\mathrm{PSL}_{3}(3)$ & $S_{4}$ & Lemma 3.1 \\
\hline
\end{tabular}

In the remaining cases (T1) and (T2) the same conclusion immediately follows from the next lemma the proof of which is based on a construction from [1] and the description of maximal subgroups of a symmetric group given in [18].

Lemma 6.1. The group $\operatorname{PSL}_{2}(q)$ is not Schur unless $q \leq 3$.

Proof. The groups $\operatorname{PSL}_{2}(2) \simeq D_{6}$ and $\operatorname{PSL}_{2}(3) \simeq A_{4}$ are Schur by Lemma 3.1. If $q=2^{k}$ and $k$ is a composite integer with a proper prime divisor $p$, then the group $P S L_{2}\left(2^{k}\right)$ contains a subgroup isomorphic to $P S L_{2}\left(2^{p}\right)$ arising in case (T3) of the Thompson theorem, so the group $P S L_{2}(q)$ with even $q$ is not Schur unless $q=2$. Since $P S L_{2}(5) \simeq A_{5}$ is also not Schur, in what follows we may assume that $q$ is odd and $q>5$.

Set $G=\operatorname{PSL}_{2}(q)$. The group $\Gamma=G_{\text {right }} \operatorname{Inn}(G)$ acts naturally on $G$, and contains a regular subgroup $G_{\text {right }}$. Therefore $\operatorname{Inv}(\Gamma)$ is a Cayley scheme over $G$ such that the basic sets of the corresponding S-ring $\mathcal{A}(\Gamma, G)$ are the conjugacy classes of $G$; the partition of $G$ with these classes is denoted by $\mathcal{C}$.

According to [1, Sections 3,4], the Cayley scheme $\operatorname{Inv}(\Gamma)$ contains a special fusion $\mathcal{X}$ of rank 4 . The definition of this scheme given there, implies that the S-ring $\mathcal{A}=\mathcal{A}(\mathcal{X})$ satisfies to the following conditions:

$\left(1^{*}\right) \operatorname{dim}_{\mathbb{Z}}(\mathcal{A})=4$, 
$\left(2^{*}\right)$ each element of $\mathcal{S}=\mathcal{S}(\mathcal{A})$ is the union of all classes of $\mathcal{C}$ of the same size,

$\left(3^{*}\right)$ if $q$ is odd, then $\mathcal{S}=\mathcal{C}$ only if $q \leq 5$.

To prove that the group $G$ is not Schur we will verify that the S-ring $\mathcal{A}$ is not schurian.

The group $\Delta=N_{\operatorname{Sym}(G)}(\Gamma)$ obviously contains a regular subgroup $G_{\text {right }}$, and hence one can form the Cayley scheme $\operatorname{Inv}(\Delta)$ and the corresponding S-ring $\mathcal{A}(\Delta, G)$. Since $\Gamma$ is normal in $\Delta$, condition $\left(2^{*}\right)$ implies that the point stabilizer $\Delta_{e}$ leaves each class of the partition $\mathcal{S}$ fixed. Moreover,

$$
\mathcal{A}(\Delta, G) \supseteq \mathcal{A} .
$$

Next, by the main theorem in 18 the group $\Delta^{\prime}:=G^{2} \cdot\left(\right.$ Out $\left.G \times C_{2}\right)$, which appears in the diagonal case (d) of that paper, is maximal in $A_{n} \Delta^{\prime}$ where $n=|G|$. Since obviously $\Delta^{\prime}$ normalizes $\Gamma=G_{\text {left }} \times G_{\text {right }}$, it follows that $\Delta^{\prime} \leq \Delta$. Thus, due to (11) we have

$$
\Delta^{\prime} \leq \Delta \leq \operatorname{Aut}(\operatorname{Inv}(\Delta)) \leq \operatorname{Aut}(\mathcal{X}) .
$$

However, by statement $\left(1^{*}\right)$ the group $\operatorname{Aut}(\mathcal{X})$ can not be 2 -transitive. So it has no a subgroup isomorphic to $A_{n}$. Therefore the maximality of $\Delta^{\prime}$ together with (12) imply that $\Delta^{\prime}=\Delta=\operatorname{Aut}(\mathcal{X})$. Thus to prove that the S-ring $\mathcal{A}$ is not schurian it suffices to verify that

$$
\mathcal{A}(\Delta, G) \neq \mathcal{A} .
$$

When $q$ is odd, this inequality immediately follows from statement $\left(3^{*}\right)$.

\section{Proof of Theorem 1.3}

Let us recall some basic facts on the Fitting subgroup $F=F(G)$ of a group $G$. By definition $F$ is the largest normal nilpotent subgroup of $G$. Suppose that $G$ is solvable. Then

$$
C_{G}(F) \leqslant F \quad \text { and } \quad G / F \prec \operatorname{Aut}(F)
$$

(e.g., [14, Theorem 6.1.3]). Moreover, $G$ has a finite Fitting height $h=h(G)$ which means by definition that there is a normal series

$$
1=F_{0}<F_{1}<\ldots<F_{h}=G
$$

where $F_{i+1} / F_{i}$ is the Fitting subgroup of $G / F_{i}$; clearly, $F_{1}=F(G)=: F$.

Turn to the proof of Theorem 1.3. The group $G$ being a Schur group must be solvable by Theorem 1.2. Moreover, by Corollary 4.3 we can assume that $G$ is not nilpotent and there is at most one prime $p$ such that a Sylow $p$-subgroup of $F$ is non-cyclic, and $p$ is equal to 2 or 3 . Then the group $G$ is metabelian by Lemmas 7.2, 7.3 and 7.4 that will be proved later. Statement (1) immediately follows from Corollary 3.5. Finally, if a Hall $\{2,3\}$-subgroup of $G$ is cyclic, then the group $F$ and by Lemma 7.2 also the group $G / F$, are direct products of Sylow subgroups. But they are cyclic by the assumtion and Theorem 3.2 This proves statement (2).

In what follows we need the following general lemma on the Fitting subgroup and its centralizer. 
Lemma 7.1. Let $H$ be a finite solvable group and $F:=F(H)=A \times B$ where $A$ and $B$ are characteristic subgroups of $H$. Then

$$
C_{H}(F)=C_{H}(A) \cap C_{H}(B) \text { and } H / F \leqslant H / C_{H}(A) F \times H / C_{H}(B) F,
$$

in particular, $H / F$ is a subgroup of a direct product of sections $K$ and $L$ of groups $\operatorname{Aut}(A)$ and $\operatorname{Aut}(B)$ respectively.

Proof. The first equality in (16) is obvious whereas the inclusion holds true by the Remak Theorem. Furthermore, $H / F C_{H}(A)$ is a factor group of $H / C_{H}(A)$ and $H / F C_{H}(B)$ is a factor group of $H / C_{H}(B)$.

In the rest of the section we always assume that $G$ is a solvable but non-nilpotent Schur group, $F=F(G)$ and $F \neq F_{2}$, see (15). We will also use the following standard notation. If $\pi$ is a set of primes, then $O_{\pi}(G)$ is the greatest normal $\pi$ subgroup of $G$; for a prime $p$ we also set $O_{p}(G)=O_{\{p\}}(G)$ and $O_{p^{\prime}}(G)=O_{\pi \backslash\{p\}}(G)$.

Lemma 7.2. Suppose that $F$ is cyclic, Then $G / F$ is abelian.

Proof. If the group $F$ is cyclic, then $\operatorname{Aut}(F)$ is abelian, so $G / F$ is abelian by (14).

Lemma 7.3. Suppose that the Sylow 3-subgroup $P$ of $F$ is non-cyclic. Then

$$
G \simeq E_{27}: C_{13} \quad \text { or } \quad G \simeq E_{9} \times\left(C_{n}: C_{m}\right)
$$

where $(n, m) \in\{(p, q),(2 p, q),(p, 2 q),(p, 4)\}, p$ and $q$ are primes greater than 3 , and $m$ divides $p-1$.

Proof. By the Burnside theorem on a basis of a $p$-group, the action of $O_{3^{\prime}}(G / F)$ on $P$ induces a group isomorphic to a $3^{\prime}$-subgroup $T$ of $\mathrm{GL}_{r}(3)$ where $r=r(P)$. On the other hand, since $F$ is not cyclic, Theorem 3.3 implies that $r=2$ or $r=3$. Therefore $\pi(T) \subseteq\{2,13\}$ and $13 \in \pi(T)$ only if $r=3$. We claim

$$
\mathrm{O}_{2}(T)=1 \text {. }
$$

Indeed, otherwise $G$ contains an involution, which normalizes a subgroup of $P$ that is isomorphic to $E_{9}$. But then $G$ contains a non-abelian section of order 18 , which is not dihedral. However, by Lemma 3.1 and Theorem 2.4 this contradicts the assumption that $G$ is a Schur group.

Without loss of generality we can assume that $G$ is not isomorphic to $E_{27}: C_{13}$. In this case we claim that

$$
r=2 \text { and } T=1 .
$$

Indeed, suppose that this is not true. Note, that if $T \neq 1$, then $13 \in \pi(T)$ by (17). Thus in any case $r=3$. So by Lemma 4.1 a Sylow 3-subgroup of $G$ is abelian. By Theorem 3.3 this implies that this subgroup coincides with $E_{27}, P=F=E_{27}$ and $O_{3}(G / F)=1$. Taking into account that $G \neq F$ we conclude that $G / F \simeq C_{13}$. Thus $G \simeq E_{27}: C_{13}$. Contradiction.

Recall that $F$ is a nilpotent Schur group and $P$ is a non-cyclic Sylow 3-subgoup. Therefore by Corollary 4.3 we have

$$
F=P \times Q
$$

where $Q$ is a cyclic $3^{\prime}$-subgroup of $F$. Since $r=2$, Theorem 3.3 implies that $n:=|Q|$ belongs to the set $\{1,2,4, p, 2 q\}$ where $p$ and $q$ are primes $\geq 5$. In fact, 
$n>2$. Indeed, otherwise the group $\operatorname{Aut}(Q)$ is trivial. Therefore $G / F$ is isomorphic to a factor group of $\operatorname{Aut}(P)$ by Lemma 7.1. Due to (18) this implies that $F_{2} / F$ is a 3-group. Moreover, this group must be trivial, because otherwise a Sylow 3 -subgroup of $F_{2}$ is normal in $G$. Thus, $F_{2}=F$. Contradiction.

It follows that $n \in\{4, p, 2 q\}$. Moreover, by Theorem 4.2 the group $P$ is either abelian or isomorphic to $M_{3^{k}}$ with $k>3$, and in the same time by Theorem 3.3 it does not contain an abelian subgroup isomorphic to $C_{3} \times C_{9}$. Thus

$$
P \simeq E_{9} .
$$

Moreover, the group $O_{3}(G / F)$ acts on $P$ trivially (otherwise $G$ contains a nonabelian subgroup of order 27, which is not Schur by Lemma 3.1). Together with (18) this shows that $F_{2} \leq C_{G}(P)$. Therefore by Lemma 7.1 we have $C_{G}(Q)=F$ and $G / F=G / C_{G}(Q) \leq \operatorname{Aut}(Q)$. Hence $G=F_{2}$, and the group $G / F$ is cyclic and acts on $P$ trivially. It follows

$$
G \simeq E_{9} \times(Q: K)
$$

for some $K \leq \operatorname{Aut}(Q)$. Thus the required statement follows from Theorem 3.3 because $E_{9} \times K$ should be a Schur group.

Lemma 7.4. Suppose that the Sylow 2-subgroup $P$ of $F$ is non-cyclic. Then $G$ is metabelian. Furthermore, if $r(P) \geq 3$, then

$$
G \simeq E_{32}: C_{31} \quad \text { or } \quad G \simeq P \times\left(C_{p}: C_{q}\right)
$$

where $P$ is one of the group $E_{8}, G_{16}, E_{16}$, the numbers $p$ and $q$ are primes, $p>3$, and $q$ divides $p-1$.

Proof. Suppose first that $F=P$. If $r(P) \geq 3$ and $P$ is abelian, then $P \simeq E_{2^{k}}$, $k \geq 3$, by Theorem 4.2. Since the group $G_{16}$ has no subgroup isomorphic to $E_{8}$, Lemma 4.1 implies that any Sylow subgroup of $G$ that contains $P$, is abelian. Due to (14) we conclude that $P$ is a Sylow 2 -subgroup of $G$. Thus the required statement follows from Lemma 3.1. If $r(P) \geq 3$ and $P$ is not abelian, then Lemma 4.1 implies that $P \simeq G_{16}$ and $P$ is again a Sylow 2-subgroup of $G$. However, this is impossible by Lemma 3.1 applied for $G / \Phi(P)$ and $P / \Phi(P) \simeq E_{8}$. Thus $r(P)=2$. If $P \simeq E_{4}$ or $P \simeq Q_{8}$, then $\operatorname{Aut}(P) \simeq S_{3}$. Due to (14) this implies that $|G| \leq 24$, and the required statement follows from Lemma 3.1. By Theorems 4.2 and 3.3 in the remaining cases $P$ is isomorphic to one of the following groups

$$
C_{2} \times C_{2^{k}}(k>1), \quad M_{2^{k}}(k>5), \quad D_{2^{k}}(k>2) .
$$

Moreover $\operatorname{Aut}(P)$ is a 2-group by [2, Theorem 34.8] and [6, Theorem 3.5]. This implies that $G$ is nilpotent in contrast to our assumption.

Let now $P$ be a proper subgroup of $F$. Then by Corollary 4.3 we have

$$
F=P \times Q
$$

where $Q$ is a nontrivial cyclic group of odd order. By Theorem 3.3 this implies that $2 \leq r \leq 4$ where $r=r(P)$. Suppose first that $r=3$ or $r=4$. Then by that theorem we can assume that

$$
P \in\left\{E_{8}, G_{16}, E_{16}\right\} \text { and } Q=C_{p}
$$

for some odd prime $p$. By the Burnside theorem on a basis the group $O_{2^{\prime}}(G / F)$ acts on $P / \Phi(P)$. If this action is not trivial, then by Lemma 3.8 the group $G$ has 
a non-abelian section of order 24 and rank 3, which is impossible by Lemma 3.1 Thus

$$
O_{2^{\prime}}(G / F) \leq C_{G}(P) F \text {. }
$$

On the other hand, if $O_{2}(G / F) \not \leq C_{G}(P) F$, then Sylow 2-subgroup of $G$ is nonabelian and includes $P$ as a proper subgroup. By Lemma 4.1 this implies that this subgroup is isomorphic to $G_{16}$ which is impossible because the latter group does not contain a subgroup isomorphic $E_{8}$. Therefore

$$
\mathrm{O}_{2}(G / F) \leq C_{G}(P) F \text {. }
$$

Together with (21) this shows that $F_{2} \leq C_{G}(P) F$. Therefore Lemma 7.1 implies that $C_{G}(Q)=F$ and $G / F=G / C_{G}(Q) \leq \operatorname{Aut}(Q)$. It follows that $G=F_{2}$ and that $G / F$ is a cyclic group acting on $P$ trivially. Applying also Lemma 3.1 and Theorem 3.3, we obtain that $G \simeq P \times\left(C_{p}: C_{q}\right)$, where $q$ and $r$ are primes, $p>3$ and $q$ divides $p-1$.

Let now $r=2$. Then by Theorem 3.3 the group $P$ is isomorphic to $E_{4}, Q_{8}$ or one of the groups in (19), and $Q=C_{p}$, or $P=E_{4}$ and $Q=C_{p q}$, where $p$ and $q$ different primes. Let us consider three cases depending on the group $P$. Below we set $K=G / F$.

Case 1: $P \not E_{4}$ and $P \not Q_{8}$. In this case $\operatorname{Aut}(P)$ is a 2-group (see above) and $Q \simeq C_{p}$ for an odd prime $p$. By (16) this implies that the group $C_{G}(Q) F / F$ is isomorphic to a section of 2 -group $G / C_{G}(P) F \prec \operatorname{Aut}(P)$. So the preimage of $C_{G}(Q) F / F$ in $G$ is a nilpotent normal subgroup. Therefore it is contained in $F$, and hence $C_{G}(Q) \leq F$. It follows that

$$
C_{G}(Q)=F \quad \text { and } \quad K \prec \operatorname{Aut}\left(C_{p}\right) .
$$

So $K$ is a cyclic group of order dividing $p-1$. This proves $G$ is metabelian, as required, whenever $P$ is abelian. So we may assume that $P$ is isomorphic to $M_{2^{k}}$, $k>5$, or $D_{2^{k}}, k>2$. If $K$ has an odd order, then

$$
C_{G}(P) F=G=P \times O_{2^{\prime}}(G),
$$

where $O_{2}^{\prime}(G)$ is a Frobenius group $C_{p}: C_{q}$ with $q>3$. In particular, $G$ is metabelian and we are done. Assume that $K$ is of even order. Then a Sylow 2 -subgroup $S$ of $G$ contains a proper normal subgroup isomorphic to $P$. Therefore

$$
S \simeq M_{2^{l}} \quad \text { or } \quad S \simeq D_{2^{l}}
$$

for some integer $l>k$. However, the first case is impossible because all proper subgroups of $M_{2^{l}}$ are abelian [2, p. 29]. In the second case, $S$ contains exactly two conjugacy classes of involutions, so index of $P$ in $S$ should be 2 and $l=k+1$. Denote by $C$ the cyclic subgroup of order $2^{k-1}$ in $P$. The subgroup $C$ is characteristic in $P$, so it is normal in $G$. Put $T=C \times Q$. Then $T$ is abelian. On the other hand, $G / T$ a central extension of the group $F / T$ of order 2 by a cyclic group $K$, so $G / T$ is abelian. It provides that $G$ is metabelian.

Case 2: $P \simeq Q_{8}$. By Theorem 4.2 none of possible Schur 2-groups contains a proper subgroup isomorphic to $P$. Therefore the group $K$ has an odd order. Moreover, by Lemma 3.1 the order of this group is not a multiple of 3 . Since the factor group $G / C_{G}(P) F$ must be isomorphic to a section of $\operatorname{Aut}\left(Q_{8}\right) \simeq S_{3}$, we conclude that

$$
G=C_{G}(P) F .
$$


By Lemma 7.1 this shows that $C_{G}(Q)=F$. Thus from Theorem 3.3 it follows that $G \simeq P \times\left(C_{p}: Z_{q}\right)$, where $p$ and $q$ are primes, $q>3$, and $q$ divides $p-1$. In particular, $G$ is metabelian.

Case 3: $P \simeq E_{4}$. In this case the group $F$ is abelian, so it sufficient to prove that so is the group $K$. If $C_{G}(P) \leq F$, then $K \leq S_{3}$. Moreover, $K$ is not isomorphic $S_{3}$, because otherwise $G / Q$ is a non-abelian and non-dihedral group of order 24, which is impossible by Lemma 3.1. Since $G$ is not nilpotent, this implies that $K$ is a cyclic group of order 3 as required. On the other hand, if $C_{G}(Q) \leq F$, then $K \prec \operatorname{Aut}(Q)$. Since $Q$ is cyclic, the group $K$ is again abelian, and we are done. Thus we can assume that

$$
C_{G}(P) \not \leq F \quad \text { and } \quad C_{G}(Q) \not \leq F .
$$

In this case $K$ contains nontrivial subgroups $K_{1}=C_{G}(P) / F$ and $K_{2}=C_{G}(Q) / F$, and by Lemma 7.1 also the direct product $M=K_{1} \times K_{2}$. Since

$$
K_{1} \prec G / C_{G}(Q) \prec \operatorname{Aut}(Q) \text { and } K_{2} \prec G / C_{G}(P) \prec S_{3} \text {, }
$$

the group $K_{1}$ is abelian whereas the group $K_{2}$ is cyclic of order 3 . Therefore the group $M$ is abelian, and we may assume that $K \neq M$. Then $|K / M|=2$ because $G / C_{G}(P) \prec S_{3}$. Moreover, the group $K_{1}$ is of odd order, for otherwise the group $G / Q$ contains a non-abelian and non-dihedral group of order 24, which is impossible by Lemma 3.1. Therefore, $M$ is a $2^{\prime}$-group. This implies that $K$ is a semidirect product of $M$ and a subgroup $N$ of order 2. Moreover, since $G / C_{G}(Q)$ is abelian, either $K$ is abelian, or $N$ acts nontrivially on $C_{G}(Q)$. But in the latter case $K$ contains a subgroup $C_{G}(Q) N$ isomorphic to $S_{3}$. Its preimage in $G / Q$ is a non-abelian and non-dihedral subgroup of order 24; a contradiction. Thus, $K$ is abelian, as required.

Acknowledgment. We would like to thank Prof. M. Muzychuk for the fruithful discussions from which Lemmas 5.3 and 6.1 arose. We are also thankful to Prof. M. Klin and Dr. S. Reichard for providing computational data on S-rings over small groups.

\section{REFERENCES}

[1] E. Bannai, Subschemes of some association schemes, J. of Algebra 144 (1991), 167-188.

[2] Y. Berkovich, Groups of prime power order. Vol. 1, de Gruyter Expositions in Mathematics, 46, Walter de Gruyter GmbH \& Co. KG, Berlin, 2008.

[3] A. E. Brouwer, A. M. Cohen, A. Neumaier, Distance-regular graphs. Ergebnisse der Mathematik und ihrer Grenzgebiete, 3. Folge, 18. Berlin etc.: Springer-Verlag, 1989.

[4] D. Jungnickel, A. Pott, and K. W. Smith, Difference Sets. In: Colbourn, C.J., Dinitz, J.H. (eds) Handbook of Combinatorial Designs, 2nd edn., pp. 419-435. Chapman, Hall/CRC, Boca Raton (2007).

[5] J. H. Conway, R. T. Curtis, S. P. Norton, R. A. Parker, and R. A. Wilson, An ATLAS of Finite Groups, Oxford University Press, Oxford, 1985.

[6] M. J. Curran, The automorphism group of a splitmetacyclic 2-group, Arch. Math., 89 (2007), $10-23$.

[7] P. Dembowski, Finite geometries, Ergebnisse der Mathematik und ihrer Grenzgebiete, Band 44 Springer-Verlag, Berlin-New York, 1968.

[8] S. Evdokimov, Schurity and separability of association schemes, Thesis (2004), 1-155.

[9] S. Evdokimov, I. Kovács and, I. Ponomarenko, Characterization of cyclic Schur groups, Algebra and Analysis, 25 (2013), 61-85.

[10] S. Evdokimov, I. Kovács, and I. Ponomarenko, On schurity of finite abelian groups, arXiv:1309.0989 [math.GR] (2013), 1-20. 
[11] S. Evdokimov, and I. Ponomarenko, Permutation group approach to association schemes, Eur. J. Comb., 30 (2009), 1456-1476.

[12] F. Fiedler, Enumeration of Cellular Algebras Applied to Graphs with Prescribed Symmetry, Master s thesis, Technische Universität Dresden, 1998.

[13] The GAP Group, GAP Groups, Algorithms, and Programming, Version 4.4.10; 2007, http://www.gap-system.org

[14] D. Gorenstein, Finite Groups, Harper \& Row, New York, 1968.

[15] A. Hanaki, and I. Miyamoto, Classification of association schemes with small number of vertices, published on web (http://kissme.shinshu-u.ac.jp/as/).

[16] A. Hanaki, Elementary Functions for Association Schemes on GAP, published on web (http://kissme.shinshu-u.ac.jp/as/gap/), 2012.

[17] W. M. Kantor, 2-transitive symmetric designs, Transactions of AMS 146 (1969), 1-28.

[18] M. W. Liebeck, Ch. E. Praeger, and J. Saxl, A classification of the maximal subgroups of the finite alternating and symmetric groups, J. of Algebra 111 (1987), 365-383.

[19] Ch. Pech, and S. Reichard, Enumerating set-orbits, in: M.Klin, G.A.Jones, A.Jurisic, M.Muzychuk, I.Ponomarenko (Eds.), Algorithmic Algebraic Combinatorics and Grobner Bases, Springer, 2009, 137-150.

[20] R. Pöschel, Untersuchungen von s-ringen insbesondere im gruppenring von p-gruppen, Math. Nachr. 60 (1974), 1-27.

[21] I. Schur, Zür theorie der einfach transitiven permutationgruppen, S.-B. Preus Akad. Wiss. Phys.-Math. Kl. (1933), 598-623.

[22] J. G. Thompson, Nonsolvable finite groups all of whose local subgroups are solvable, Bull. Amer. Math. Soc., 74 (1968), 383-437.

[23] R. Wilson, The finite simple groups, Graduate Texts in Mathematics, no. 251, Springer, 2009.

[24] H. Wielandt, Finite permutation groups, Academic press, New York - London, 1964.

[25] H. Wielandt, Permutation groups through invariant relations and invariant functions, Lect. Notes Dept. Math. Ohio St. Univ., Columbus, 1969.

Steklov Institute of Mathematics at St. Petersburg, Russia

E-mail address: inp@pdmi.ras.ru

Sobolev Institute of Mathematics and Novosibirsk State University, Novosibirsk, RUsSiA

E-mail address: vasand@math.nsc.ru 